\title{
APLICACIÓN DE LOS SISTEMAS DE INFORMACIÓN GEOGRÁFICA AL ESTUDIO DE LA DISTRIBUCIÓN URBANA DE LA TERCERA EDAD. EL CASO DE SEVILLA
}

Rosa JORDÁ BORRELL*

Ángel Luis LUCENDO MONEDERO**

\section{INTRODUCCIÓN. LOS OBJETIVOS DEL PROYECTO}

El envejecimiento demográfico es un fenómeno geográfico en emergencia que puede llegar a originar serios problemas macroeconómicos estructurales a nuestra sociedad. Pues este grupo de población se incrementa de forma cuantitativa mientras se deteriora cualitativamente por la falta de recursos sociales y asistenciales que frenen la situación de desigualdad y marginación que padecen frente al resto de la sociedad. Por todo ello se hace necesario abordar cuanto antes políticas dirigidas a mejorar la calidad de vida de la gente mayor que estén basadas en estudios que definan su situación espacial y social en aquellos lugares donde su presencia es más numerosa, como es el caso de las grandes ciudades.

Tradicionalmente la Geografía, en el ámbito de la demografía, se ha interesado por el crecimiento diferencial de los lugares, es decir, por investigaciones ligadas más al comportamiento de los jóvenes que al comportamiento específico de otros grupos poblacionales. Sin embargo, actualmente la Geografía, como ciencia aplicada a la identificación y resolución de problemas espaciales, contribuye a la Demografía con otro tipo de estudios orientados a analizar la distribución y caracterización del envejecimiento y las relaciones entre espacio que integra el ser social del anciano y sus necesidades de servicios sociales ${ }^{1}$. Hasta ahora, la aplica-

* Catedrática de Análisis Geográfico Regional. Universidad de Sevilla.

** Profesor Asociado a la misma área.

1. La concentración de la población en las grandes ciudades de nuestro entorno geográfico ha provocado serias 
ción de métodos de análisis geográfico para solucionar los problemas que plantean este tipo de fenómenos vienen centrando su interés, sobre todo, en la distribución geográfica del envejecimiento; la descripción y explicación de modelos; y en la localización de recursos y desarrollo efectivo de los servicios destinados a los ancianos (ROWLES, 1986).

El presente trabajo de investigación se propone identificar áreas urbanas homogéneas que tengan el mismo tipo de población anciana en el municipio de Sevilla por medio de la aplicación, por un lado, de una serie de métodos estadísticos que nos permitan caracterizar el envejecimiento demográfico y físico de los ancianos de esta ciudad; y por otro, a partir del estudio de la distribución geográfica de los distintos tipos de mayores que puedan ayudarnos a la identificación y localización de los mismos.

El objetivo principal de este trabajo de investigación es el estudio de la distribución de las distintas tipologías de ancianos en el municipio de Sevilla, es decir, delimitar áreas homogéneas que tengan el mismo tipo de población de la tercera edad. Como paso previo es necesario identificar los distintos grupos de ancianos que existían en Sevilla en 1991, grupos definidos por las mismas características demográficas y de vivienda. Podemos decir, pues, que el objetivo de este trabajo es doble: identificar los principales rasgos socioeconómicos, sociodemográficos y de viviendas que definen los distintos tipos de ancianos, y delimitar áreas urbanas compuestas por los mismos grupos de ancianos.

El estudio de la caracterización de la población de la tercera edad en el municipio de Sevilla conlleva, igualmente, el análisis de la distribución espacial de las personas ancianas en nuestras ciudades. Desde el principio en este proyecto nos ha interesado el estudio simultáneo tanto de los aspectos o estadísticos, como de los geográficos de la tercera edad en el municipio de Sevilla, pues es evidente que ambos se interrelacionan en un modelo cartográfico, que según BERRY (1986) y TOMLIN (1990), comprende el análisis de las variables temáticas y de las características espaciales de los objetos donde se han observado dichas variables. Es por ello que, como objetivo secundario de este trabajo de investigación, también pretendemos realizar una cartografía temática que refleje la distribución espacial de las distintas variables analizadas sobre la población anciana.

La cartograffa de toda esta de información geográfica ha sido posible gracias a la utilización del programa ARCVIEW v.3.0a GIS, un sistema de información geográfica (S.I.G.). Y es que las herramientas S.I.G. permiten la gestión, manipulación y visualización de la información contenida en bases de datos temáticas y gráficas por medio de la georreferenciación. Se entiende por tal concepto al hecho de dotar a los elementos geográficos de una referencia locacional (ROBINSON

repercusiones sociales y económicas cuyos principales efectos son el deterioro de los servicios, los problemas de circulación y los fenómenos de infravivienda y deterioro físico de los centros históricos provocando la aparición de grupos marginales entre los que se encuentra los ancianos. 
et AL., 1987), o al proceso por el cual se determina la localización espacial de cada objeto geográfico (BOSQUE SENDRÁ, 1992). Los sistemas de información geográfica permiten ésto uniendo las bases de datos alfanuméricas y las gráficas, lo que da lugar a una cartografía abundante, tanto como variables contiene las fuentes utilizadas. El conjunto de los mapas originados a partir de las distintas variables demográficas y de viviendas han constituido un Atlas de la Población Anciana del Municipio de Sevilla, documento que se recoge en un volumen aparte.

Una vez georreferenciadas las bases de datos con el programa ARCVIEW, los distintos mapas obtenidos se han representado sobre una cartografía digital de la ciudad Sevilla denominada ARCÓPOLIS. El callejero digital Arcópolis es un conjunto de datos geográficos que posee tres capas de información cartográfica de la ciudad de Sevilla: la división administrativa (secciones censales, distritos y término municipal), los ejes de calle y el fondo urbano. También se ha representado los grupos de ancianos que hemos clasificados por medio del análisis cluster al nivel de sección censal. Esta cartografía nos ofrece, a la escala adecuada, un mapa de la distribución y localización de los grupos de ancianos en el municipio de Sevilla.

\section{LOS ANCIANOS DE SEVILLA COMO SUJETO DE ESTUDIO. EL ÁMBITO ESPACIAL}

Los grupos de población sujetos de estudio son las personas ancianas que había en Sevilla en 1991. Se ha tomado este año por ser el último en el que se realizó un Censo oficial, tal y como se explica en el siguiente capítulo. El criterio que establece el paso de una persona a la categoría de anciano es fundamentalmente la edad de retiro o jubilación, y secundariamente el deterioro del estado de salud (LÓPEZ JIMÉNEZ, 1993). Sin embargo ni una ni otra situaciones son exclusivas de las personas de 65 años de edad, sino que en la actualidad estos criterios afectan a un número elevado de personas entre 60 y 64 años (sobre todo el de la jubilación). Por tanto, desde el punto de vista sociodemográfico el umbral de las población anciana está en los 65 años de edad, mientras que para aspectos relacionados con la actividad laboral la población de 60 y más años de edad resulta un grupo más adecuado a pesar de las dificultades comparativo-metodológicas que esto supone. Por ello hemos seleccionado como sujeto de estudio a:

i) los ancianos propiamente dichos, es decir, según el criterio sociodemográfico la población mayor de 65 años.

ii) la población preanciana, que engloba a aquellas personas según la edad de jubilación, es decir, entre 60 y 64 años. También es conveniente ampliar el estudio a este grupo con idea de incorporar al análisis de la población de la 
tercera edad a los que serán futuros ancianos ya que al trabajar con datos de 1991 este grupo, cinco años después, ya tendrá más de 65 años y formarán parte del grupo anterior de ancianos propiamente dicha que hemos delimitado en función de la edad.

En 1991 vivían en Sevilla 114969 ancianos (población de derecho), lo que supone un incremento aproximado del 1,5\% de la población anciana desde 1986 (Cuadro I). Este dato supone un $16,79 \%$ del total de las personas residentes en Sevilla en 1991, lo que pone de manifiesto la importancia cuantitativa y cualitativa de las personas ancianas en Sevilla, más si cabe ante la falta de recursos sociales y asistenciales que aborden la situación de desigualdad entre la gente mayor puesta de manifiesto por los citados estudios.

\section{CUADRO I \\ EVOLUCIÓN DE LA POBLACIÓN DE DERECHO MAYOR DE 65 AÑOS EN LA CIUDAD DE SEVILLA}

\begin{tabular}{lrrrrrrr}
\hline & \multicolumn{2}{c}{ Año 1981} & \multicolumn{2}{c}{ Año 1986} & \multicolumn{2}{c}{ Año 1991} \\
\cline { 2 - 7 } & Población & $\%$ & Población & $\%$ & Población & $\%$ \\
\hline Población Total & 645.817 & $100,00 \%$ & 651.084 & $100,00 \%$ & 683.028 & $100,00 \%$ \\
Población > 65 Años & 50.062 & $7,75 \%$ & 67.778 & $10,41 \%$ & 80.246 & $11,75 \%$ \\
$\quad$ Hombres & 21.885 & $43,72 \%$ & 24.340 & $35,91 \%$ & 29.793 & $37,13 \%$ \\
- Mujeres & 28.177 & $56,28 \%$ & 43.438 & $64,09 \%$ & 50.453 & $62,87 \%$ \\
\hline
\end{tabular}

En cuanto al ámbito espacial de análisis para la delimitación de áreas homogéneas de la población anciana del municipio de Sevilla, este ha sido la sección urbana o sección censal. Se puede considerar a la sección censal como una verdadera unidad espacial de análisis. Además la sección censal es la unidad espacial más reducida en la que se organiza la información. De hecho, como veremos posteriormente, información proveniente del Censo de Población y Viviendas de 1991 utilizada posee este nivel de desagregación. Aunque en algunos estudios se han utilizado unidades espaciales mayores que las secciones censales ${ }^{2}$, los estudios de

2. Existen tres niveles en Ias unidades espaciales en los que se puede recoger la información: secciones censales, barrios y distritos. También hay casos, como es el estudio sobre la diferenciación residencial en el espacio urbano de Sevilla realizado por BASULTO y ARIAS, basados en «áreas morfológicas» de la Gerencia de Urbanismo del Ayuntamiento de la ciudad construidas a partir de criterios como evitar dividir barrios de morfología definida, respetar las arterias principales y/o sistemas generales que delimitan dichas áreas, o que los límites de las áreas coincidan con las secciones padronales con objeto de no dividir barrios culturalmente consolidados. Junto a estos criterios morfológicos se añade otro de demográfico: que la población del área no supere las 60.000 personas (BASULTO, y ARIAS, 1989). 
este carácter que se han realizado a partir de la información recogida en censos o padrones tienen mayor exactitud y aproximación cuando se utiliza como unidad de observación la sección censal (OCAÑA OCAÑA, 1988). Por tanto, la unidad territorial recomendada para este estudio es la sección urbana o sección censal. La ciudad de Sevilla se articula en 442 secciones, agrupadas en 6 distritos.

Las secciones censales, sin embargo, presentan una peculiaridad en cuanto al análisis geográfico de los ancianos y la interpretación estadística de sus características, ya que la sección censal es tratada como una unidad espacial cuando en realidad está formada por un conjunto de individuos. Surge aquí el problema de determinar cuáles de las distintas características de esos individuos que coexisten en cada sección es la que le da valor o la caracteriza. Por tanto, realmente estamos caracterizando las distintas secciones censales en función de las variables que más predominan de los ancianos que viven en ellas.

\section{LAS BASES DE DATOS UTILIZADAS}

En cuanto a las variables que hemos tenido en cuenta para la delimitación de áreas homogéneas de la población anciana del municipio de Sevilla, se seleccionaron las variables sociodemográficas y de viviendas disponibles del Censo de Población y Viviendas de 1991, el último realizado y, por tanto, el más reciente en el tiempo. El uso de esta fuente de información garantiza la fiabilidad y la calidad de los datos. Los datos obtenidos estaban disponibles al nivel de desagregación deseado, es decir y como ya comentamos, al nivel de sección censal. Las variables seleccionadas son de dos tipos:

a) Demográficas. Todas las tablas de población se refieren a población de derecho de 60 y más años, excepto la primera tabla que también presenta la población de hecho de 60 y más años por sexo. Las demás tablas contienen variables y categorías (por sexo) referentes a:

- Población de derecho de 60 y más años según grupos quinquenales de edad y estado civil.

- Población de derecho de 60 y más años según el lugar de procedencia (Municipio, Provincia, Comunidad Autónoma, España y del Extranjero).

- Población de derecho de 60 y más años según el nivel de instrucción (Analfabetos, Sin Estudio, Primer Grado, Segundo Grado y Tercer Grado).

- Población de derecho de 60 y más años en relación con la actividad (Activos, Ocupados, Parados e Inactivos) la rama de actividad (por los distintos sectores) y la situación profesional (Empresarios que emplean, Empresarios que no emplean, Cooperativistas, Ayuda Familiar, Trabajador Fijo, Trabajador Eventual y Otros). 
- Población de derecho de 60 y más años según la clase de vivienda (Principal, No Principal, Alojamiento y Colectiva).

b) Para las tablas requeridas de viviendas se han seleccionado todas aquellas viviendas en Sevilla capital, en las que al menos habite una persona de 60 o más años. Cada una de estas tablas llevan el filtro de clase de vivienda requerido por la variable que se tabula. Las tablas poseen datos de:

- Viviendas familiares según clase de vivienda (Principal, No Principal, Alojamientos y Colectivos).

- Viviendas familiares por el número de viviendas del edificio (con 1, con 2, y con 3 y más viviendas).

- Viviendas familiares según la superficie (Menos de $30 \mathrm{~m}^{2}$, de 30 a $60 \mathrm{~m}^{2}$, De 61 a $90 \mathrm{~m}^{2}$, De 91 a $120 \mathrm{~m}^{2}$, y Más de $120 \mathrm{~m}^{2}$ ).

- Viviendas familiares según el número de habitaciones (desde 1 a 6 y más Habitaciones).

- Viviendas principales según número de ocupantes (desde 1 a 5 y más Ocupantes ancianos).

- Viviendas familiares según el año de construcción (Antes de 1941, De 1941 a 1960, De 1961 a 1980 y De 1981 a 1991).

- Viviendas principales según el régimen de tenencia (Propiedad, Alquiler, Okos).

- Viviendas familiares según sus instalaciones y servicios (combustible calefacción, calefacción, teléfono, gas, energía eléctrica, refrigeración, agua corriente, agua caliente, retrete, baño o ducha).

El tratamiento de las bases de datos se hizo con ayuda de los programas informáticos estadísticos STATGRAPHICS v.1.4, el CSS v.5 y el SPSS v.6.0.1, ambos bajo Windows 95, además de la hoja de cálculo Microsoft EXCEL versión 5.0 .

\section{CARACTERIZACIÓN E IDENTIFICACIÓN DE LOS GRUPOS DE MAYORES DE SEVILLA}

El estudio de la caracterización de los ancianos es, como hemos comentado, el paso previo a la delimitación de áreas homogéneas. Para conseguir este objetivo, y ante al amplio número de variables que definen a la población anciana, es necesario realizar un análisis estadístico de tipo descriptivo de las que tiene por objeto resumir, con unos pocos datos, las principales características del conjunto de la población anciana el municipio de Sevilla. Además, esos datos sirven de base para 
la realización de la cartografía sobre la distribución de los ancianos. Sin embargo, el análisis descriptivo es del todo insuficiente para hallar áreas homogéneas con población de la tercera edad de las mismas características, ya que sólo nos ofrece una visión resumida del conjunto de los ancianos (Cuadro II).

\section{CUADRO II}

SECCIÓN CENSAL MEDIA DEL MUNICIPIO DE SEVILLA SEGÚN LA POBLACIÓN ANCIANA Y LAS VIVIENDAS EN LAS QUE RESIDEN (VALORES MEDIOS)

\begin{tabular}{|c|c|}
\hline $\begin{array}{c}\text { POBLACION } \\
\text { TOTALES } \\
260 \text { ANCIANOS }\end{array}$ & $\begin{array}{c}\text { VIVIENDAS } \\
\text { TOTALES } \\
177 \text { VIVIENDAS }\end{array}$ \\
\hline $\begin{array}{c}\text { EDAD } \\
140 \text { ANCIANOS MENORES DE } 70 \text { AÑOS } \\
120 \text { ANCIANOS MAYORES DE } 70 \text { AÑOS } \\
\text { ESTADO CIVIL } \\
\text { SOLTEROS: } 82 \text { HOMBRES - } 67 \text { MUJERES } \\
\text { VIUDOS: } 11 \text { HOMBRES - } 65 \text { MUJERES } \\
\text { ORIGEN } \\
\text { DE SEVILLA: } 36 \text { HOMBRES - } 57 \text { MUJERES } \\
\text { DE LA PROVINCIA: } 20 \text { HOMBRES - } 32 \text { MUJERES } \\
\text { DE ANDALUCIA: } 25 \text { HOMBRES - } 40 \text { MUJERES } \\
\text { DE ESPAÑA: } 16 \text { HOMBRES - } 25 \text { MUJERES } \\
\text { EXTRANJEROS: I HOMBRE - } 2 \text { MUJERES } \\
\text { INSTRUCCIÓN } \\
\text { ANALFABETOS: } 4 \text { HOMBRES -19 MUJERES } \\
\text { SIN ESTUDIOS: } 41 \text { HOMBRES - } 78 \text { MUJERES } \\
\text { PRIMER GRADO: } 30 \text { HOMBRES - } 45 \text { MUJERES } \\
\text { SEGUNDO GRADO: } 16 \text { HOMBRES - } 14 \text { MUJERES } \\
\text { TERCER GRADO: } 10 \text { HOMBRES - } 4 \text { MUJERES } \\
\text { ACTIVIDAD } \\
\text { ACTIVOS: } 19 \text { HOMBRES - } 5 \text { MUJERES } \\
\text { OCUPADOS: } 16 \text { HOMBRES - } 4 \text { MUJERES } \\
\text { EN PARO: } 3 \text { HOMBRES - } 1 \text { MUJER } \\
\text { INACTIVOS: } 82 \text { HOMBRES - 256 MUJERES } \\
\text { SECTORES } \\
\text { INDUSTRIA: } 2 \text { HOMBRES - } 0,3 \text { MUJERES } \\
\text { SERVICIOS: } 11 \text { HOMBRES - MUJERES } \\
\text { SITUACIÓN PROFESIONAL } \\
\text { TMPRESARIOS: } 4 \text { HOMBRES - 0,7 MUJERES } \\
\text { TRABAJADORES: } 10 \text { HOMBRES - } 3 \text { MUJERES }\end{array}$ & $\begin{array}{c}\text { EDIFICIOS } \\
\text { CON I VIVIENDA: } 23 \\
\text { CON } 2 \text { VIVIENDAS: } 5 \\
\text { CON } 3 \text { VIVIENDAS: } 149 \\
\text { SUPERFICIE } \\
\text { MENOS } 30 \mathrm{M}^{2}: 5 \\
\text { ENTRE } 30 \text { Y } 60 \mathrm{M}^{2}: 83 \\
\text { ENTRE } 61 \text { Y } 90 \mathrm{M}^{2}: 78 \\
\text { ENTRE } 91 \text { Y } 120 \mathrm{M}^{2}: 25 \\
\text { MAS DE } 120 \mathrm{M}^{2}: 18 \\
\text { HABITACIONES } \\
\text { CON I HABITACIÓN: I } \\
\text { CON } 2 \text { HABITACIONES } 7 \\
\text { CON } 3 \text { HABITACIONES: } 33 \\
\text { CON } 4 \text { HABITACIONES: } 54 \\
\text { CON } 5 \text { HABITACIONES: } 49 \\
\text { CON 16 Y MAS HABITACIONES: } 32 \\
\text { OCUPANTES } \\
\text { CON } 1 \text { OCUPANTE: } 103 \\
\text { CON } 2 \text { OCUPANTES: } 69 \\
\text { CON } 3 \text { Y MAS OCUPANTES: } 5 \\
\text { AÑ CONSTRUCCIÓN } \\
\text { ANTES 1941: } 22 \\
\text { ENTRE 1941 Y 1960: } 42 \\
\text { ENTRE 1961 Y 1980: } 102 \\
\text { ENTRE 1981 Y 1991: } 10 \\
\text { TENENCIA } \\
\text { EN PROPIEDAD: } 128 \\
\text { EN ALQUILER: } 27 \\
\text { OTROS: } 22\end{array}$ \\
\hline
\end{tabular}


Para poder identificar grupos de ancianos con las mismas características es necesario saber cuales son las variables que asocian para diferenciar unos grupos de otros. Por ello se plantea la necesidad de aplicar métodos estadísticos más avanzados que muestren las relaciones entre variables y categorías de la población anciana de Sevilla como son: el análisis detallado de todas las correlaciones lineales entre las variables demográficas y de viviendas (para saber la fuerza con que dos variables están relacionadas); la aplicación del análisis factorial (que va a poner de manifiesto la interdependencia de las variables analizadas en unos pocos factores que explican las interrelaciones entre las variables; y la aplicación del análisis cluster (que permite formar, a partir de un conjunto de casos u observaciones, sucesivos grupos homogéneos).

\section{a) Análisis de las principales correlaciones entre variables}

Analizando la relaciones entre las variables demográficas y de viviendas sobre las personas de 60 y más años en Sevilla en 1991, se constata que el número de ocupantes por vivienda en las que al menos reside una persona mayor es la única variable de éste tipo que presenta unas correlaciones significativas con algunas de las características demográficas de este grupo de población (Cuadro III). Así con respecto a la variable estado civil de los ancianos, vemos que existe una casi completa asociación entre los ancianos SOLTEROS y aquellas viviendas OCUPADAS POR 2 ANCIANOS, y entre los ancianos VIUDOS y aquellas viviendas con 1 OCUPANTE ANCIANO. También es muy importante la relación SOLTEROS-UN OCUPANTE y la de VIUDOS-DOS OCUPANTES. En cuanto a aquellas viviendas con 3 Y MÁS OCUPANTES, presenta un menor grado de asociación que las anteriores viviendas y tiene una correlación igual con los SOLTEROS y los CASADOS, y menor con los VIUDOS.

Con la variable edad de los ancianos tenemos una correlación decreciente entre las viviendas con 1 y 2 OCUPANTES y la edad de esos ancianos, de forma que a menor edad (los ancianos de edades comprendidas entre 60 y 80 AÑOS mayor correlación; y a mayor edad MAS DE 80 AÑOS), menor relación. Las viviendas con 3 Y MÁS OCUPANTES mantienen una relación creciente con la edad (de menor intensidad que las anteriores excepto para los ancianos con MAS DE 85 AÑOS), de forma que a menor edad, menor relación.

Con la variable nivel de instrucción de los ancianos la relación se establece cuanto menor es el nivel de estudios de los mayores, más relación con las viviendas con menos ocupantes (ancianos ANALFABETOS hasta 1 GRADO con 1 Y 2 OCUPANTES); y cuanto mayor es el grado de instrucción, las viviendas donde residen al menos un anciano tienen más ocupantes (ancianos con 1, 2 y 3 GRADO con las viviendas con 3 Y MÁS OCUPANTES). 
CUADRO III

PRINCIPALES CORRELACIONES ENTRE VARIABLES DEMOGRÁFICAS Y DE VIVIENDA

Con 1 Ocup. Con 2 Ocup. Con 3 Ocup. Con 3 Viviendas

\begin{tabular}{lcccc}
\hline Casados & 0,41 & 0,50 & 0,54 & 0,40 \\
Solteros & 0,78 & 0,98 & 0,54 & 0,77 \\
Viudos & 0,96 & 0,79 & 0,49 & 0,72 \\
Separados-Divorciados & 0,48 & 0,33 & 0,29 & 0,34 \\
& & & & \\
60-64 Años & 0,84 & 0,90 & 0,51 & 0,73 \\
65-69 Años & 0,86 & 0,92 & 0,53 & 0,78 \\
70-74 Años & 0,85 & 0,87 & 0,55 & 0,76 \\
75-79 Años & 0,78 & 0,81 & 0,60 & 0,69 \\
80-85 Años & 0,66 & 0,65 & 0,57 & 0,53 \\
Más de 85 Años & 0,56 & 0,57 & 0,58 & 0,45 \\
& & & & \\
Activos & 0,54 & 0,59 & 0,64 & 0,53 \\
Ocupados & 0,47 & 0,54 & 0,63 & 0,49 \\
Parados & 0,51 & 0,45 & 0,20 & 0,36 \\
Inactivos & 0,91 & 0,94 & 0,57 & $0,7 \mathrm{~S}$ \\
& & & & \\
Industria & 0,45 & 0,47 & 0,38 & 0,44 \\
Servicios & 0,40 & 0,45 & 0,58 & 0,43 \\
& & & & \\
Analfabetos & 0,30 & 0,184 & $-0,13$ & 0,01 \\
Sin Estudios & 0,75 & 0,70 & 0,22 & 0,52 \\
1 Grado & 0,69 & 0,78 & 0,55 & 0,74 \\
2 Grado & 0,39 & 0,52 & 0,65 & 0,51 \\
3 Grado & 0,22 & 0,33 & 0,54 & 0,32 \\
\hline
\end{tabular}

Respecto de la actividad y los sectores de ocupación cabe decir que los ancianos ACTIVOS (y por tanto OCUPADOS) tienen una mayor relación cuanto mayor es la ocupación en las viviendas de los ancianos, y al contrario ocurre con el PARO. La correlación más fuerte se da entre las viviendas con menos ocupantes (UN OCUPANTE y, sobre todo, DOS) y los INACTIVOS, decreciendo ésta con las viviendas con 3 Y MÁS OCUPANTES.

En cuanto a los sectores de mayor ocupación de los ancianos, SERVICIOS e INDUSTRIA, las correlaciones con el número de residentes por vivienda aumenta cuando pasamos de I a 2 OCUPANTES, para luego seguir dos caminos muy distintos: mientras la correlación entre los SERVICIOS y las viviendas con $3 \mathrm{Y}$ MÁS OCUPANTES sigue aumentando, la de éstas con los ocupados en INDUSTRIA disminuye. 
Por último resaltar la existencia de importantes correlaciones entre los variables demográficas concretas de los ancianos y otra característica de las viviendas donde éstos residen: los edificios con 3 Y MAS VIVIENDAS. Esta variable presenta correlación con determinados estados civiles (los SOLTEROS y VIUDOS), con la actividad (más concretamente, con los INACTIVOS), y con un nivel de instrucción intermedio (con los 1 GRADO). Sin embargo, los edificios con $3 \mathrm{Y}$ MÁS VIVIENDAS tienen una relación más marcada con las distintas edades de los ancianos: a menor edad (de 60 A 74 AÑOS) mayor relación con este tipo de edificios.

\section{b) Análisis Factorial}

El Análisis Factorial nos permitió reducir el alto número de variables de población y vivienda de las personas mayores del municipio de Sevilla a sólo seis factores seleccionados en función del porcentaje de la varianza total que representan y a partir del gráfico de sedimentación. De estos seis factores, los dos primeros son de tipo demográfico y de vivienda, y los cuatro restantes exclusivamente de vivienda (aunque en el último se detectó la presencia de una variable de tipo demográfica). El programa STATGRAPHICS no nos permitía analizar la totalidad de las mismas en su conjunto sino sólo un máximo de 32 variables. Esto nos obligó a realizar diversos análisis factoriales para determinar qué 32 variables se introducirían en el definitivo. Estos análisis previos se llevaron a cabo con otros programas estadísticos, concretamente el CSS v.5 y el SPSS v.6.0.1, ambos bajo Windows 95 . En la selección de las 32 variables finales se tuvo en cuenta las comunalidades de las mismas, es decir, la cantidad de varianza que una variable original compartía con las demás variables en cada análisis. En la Tabla 4 que muestra la matriz factorial rotada, pueden verse además del porcentaje de la varianza explicada por cada factor y el coeficiente de saturación de cada variable, la comunalidad estimada de cada variable. De las 32 finalmente seleccionadas sólo hay dos que tienen una comunalidad por debajo del 50\%, (No Electricidad y Antes de 1941), incluidas porque consideramos, a priori, importantes en la caracterización de la tercera edad de Sevilla.

El primero de los factores explica el $31.2 \%$ de la varianza total y está compuesto por variables de tipo sociodemográficas (edad, procedencia y nivel de instrucción), de vivienda (tamaño, ocupantes) y socioeconómicas (inactividad), según se desprende de los análisis previos. Se puede observar como este factor se asocia a aquellos ancianos cuyas características demográficas son: origen cercano (del municipio o de la provincia), sin instrucción o con un nivel de instrucción bajo ( sin estudios o el primer grado); y a características de vivienda de pequeño núcleo familiar (uno o dos ocupantes ancianos) y tamaño pequeño-mediano (de 30 
CUADRO IV

COMUNALIDADES Y MATRIX FACTORIAL ROTADA

\begin{tabular}{|c|c|c|c|c|c|c|c|}
\hline Variables & $\begin{array}{l}\text { Comu- } \\
\text { nalidad }\end{array}$ & $\begin{array}{c}\text { Factor } \\
1\end{array}$ & $\begin{array}{c}\text { Factor } \\
2\end{array}$ & $\begin{array}{c}\text { Factor } \\
3\end{array}$ & $\begin{array}{c}\text { Factor } \\
4\end{array}$ & $\begin{array}{c}\text { Factor } \\
5\end{array}$ & $\begin{array}{c}\text { Factor } \\
6\end{array}$ \\
\hline Entre 70 y 74 años & 0,88 & 0,86 & 0,34 & 0,01 & $-0,01$ & 0,05 & $-0,17$ \\
\hline Entre 80 y 85 años & 0,71 & 0,66 & 0,45 & $-0,10$ & $-0,17$ & 0,02 & 0,09 \\
\hline Sin estudios & 0,77 & 0,89 & $-0,22$ & $-0,02$ & 0,09 & 0,01 & 0,04 \\
\hline Grado uno & 0,64 & 0,73 & 0,38 & $-0,04$ & 0,04 & $-0,01$ & $-0,10$ \\
\hline Del municipio & 0,86 & 0,84 & 0,22 & $-0,02$ & $-0,10$ & 0,01 & 0,00 \\
\hline De la provincia & 0,69 & 0,76 & 0,01 & $-0,03$ & 0,24 & 0,01 & 0,02 \\
\hline De 30 a $60 \mathrm{mts}$. & 0,84 & 0,73 & $-0,32$ & 0,16 & $-0,16$ & 0,04 & 0,03 \\
\hline 1 Ocupante & 0,85 & 0,88 & 0,25 & $-0,04$ & 0,06 & 0,01 & 0,05 \\
\hline 2 Ocupantes & 0,90 & 0,86 & 0,36 & 0,03 & 0,15 & 0,00 & 0,06 \\
\hline De 1941 a 1960 & 0,80 & 0,66 & 0,07 & 0,12 & $-0,22$ & 0,00 & 0,08 \\
\hline Grado dos & 0,91 & 0,20 & 0,92 & $-0,01$ & $-0,03$ & $-0,03$ & $-0,03$ \\
\hline Grado tres & 0,93 & $-0,03$ & 0,94 & 0,03 & $-0,07$ & $-0,02$ & 0,16 \\
\hline Activos & 0,85 & 0,36 & 0,85 & $-0,01$ & 0,01 & $-0,01$ & $-0,04$ \\
\hline De España & 0,85 & 0,46 & 0,78 & 0,02 & 0,08 & $-0,02$ & 0,03 \\
\hline Servicios & 0,78 & 0,22 & 0,85 & $-0,03$ & $-0,07$ & $-0,01$ & 0,03 \\
\hline De 90 a 121 mts. & 0,50 & $-0,02$ & 0,70 & $-0,08$ & 0,04 & $-0,02$ & 0,03 \\
\hline Más $121 \mathrm{mts}$. & 0,81 & $-0,04$ & 0,87 & 0,02 & $-0,10$ & 0,02 & 0,02 \\
\hline 3 Ocupantes & 0,59 & 0,41 & 0,63 & $-0,04$ & $-0,10$ & $-0,04$ & 0,02 \\
\hline No teléfono & 0,80 & $-0,08$ & 0,03 & 0,86 & 0,07 & $-0,20$ & $-0,03$ \\
\hline No agua cal. & 0,74 & 0,05 & $-0,04$ & 0,83 & 0,11 & 0,02 & 0,01 \\
\hline No electricidad & 0,43 & $-0,06$ & $-0,03$ & 0,60 & $-0,06$ & 0,09 & $-0,08$ \\
\hline No ducha & 0,52 & 0,10 & 0,01 & 0,69 & 0,00 & $-0,18$ & $-0,08$ \\
\hline Cocina & 0,68 & 0,09 & $-0,08$ & 0,61 & 0,01 & 0,53 & $-0,11$ \\
\hline De 191 a 1980 & 0,67 & 0,20 & 0,09 & $-0,01$ & 0,77 & $-0,02$ & $-0,01$ \\
\hline De 60 a $90 \mathrm{mts}$. & 0,71 & 0,34 & 0,01 & $-0,18$ & 0,45 & $-0,05$ & $-0,04$ \\
\hline Propiedad & 0,78 & 0,56 & 0,37 & 0,01 & 0,59 & $-0,02$ & $-0,08$ \\
\hline Refriger. & 0,60 & 0,13 & $-0,05$ & $-0,01$ & $-0,04$ & 0,89 & $-0,01$ \\
\hline Cal. indiv. & 0,83 & 0,07 & $-0,03$ & $-0,04$ & 0,02 & 0,82 & $-0,18$ \\
\hline Gas & 0,80 & $-0,15$ & 0,04 & $-0,08$ & $-0,06$ & 0,58 & 0,01 \\
\hline Menos de $30 \mathrm{mts}$. & 0,68 & 0,34 & 0,01 & $-0,18$ & 0,45 & $-0,05$ & 0,76 \\
\hline Antes 1941 & 0,37 & 0,21 & 0,27 & $-0,17$ & $-0,17$ & 0,02 & 0,67 \\
\hline Alquiler & 0,82 & 0,31 & 0,31 & $-0,13$ & $-0,13$ & 0,03 & 0,67 \\
\hline \% Var. explicada & - & 31,2 & 13,8 & 9,2 & 7,8 & 6,4 & 5,2 \\
\hline
\end{tabular}


a 60 metros), que han sido construidas entre los años 1941 y 1960. En los análisis previos realizados para seleccionar las 32 variables finales vimos, además, que factor está influido por el tamaño de las secciones censales (numero de ancianos y de viviendas), por la variable edad (entre 60 y 75 años), por la condición de solteros o viudos, por la inactividad y por las viviendas entre 3 y 4 habitaciones. Por tanto, podemos decir que este factor engloba características de ancianos no muy mayores, con un núcleo familiar pequeño, y de niveles medios según la instrucción-actividad y el tipo de vivienda.

El segundo factor explica un porcentaje de la varianza bastante inferior al anterior $(13,8 \%)$ y se ve afectado también por variables de tipo sociodemográficas (edad, procedencia y nivel de instrucción), socioeconómicas (actividad) y de vivienda (tamaño, ocupantes). Más concretamente, está relacionado con las variables de población anciana procedente de España, con un nivel de instrucción medio-alto (segundo y tercer grado), aún activa laboralmente y en el sector servicios. En cuanto a las características de vivienda asociadas a este segundo factor destaca un núcleo familiar amplio (tres y más ancianos) y, en consecuencia, un tamaño grande de la misma (más de 120 metros). En los análisis previos vimos además que factor mostraba una alta correlación positiva con los ancianos de mayor edad (de 80 y más años), con la condición de casados o viudos, y que sus viviendas tenían más de 5 habitaciones. Por tanto, podemos decir que este factor engloba características de ancianos muy mayores, muy formados, con un núcleo familiar amplio, y de niveles altos según la instrucción-actividad y el tipo de vivienda.

El tercer factor explica el 9,2\% de la varianza total y está compuesto por variables relativas a las instalaciones y servicios de las viviendas que ocupan los ancianos de Sevilla. Se puede observar que las variables asociadas a este factor son aquellas relativas a la falta de una serie de equipamientos elementales en las viviendas, mucho más necesarios, si cabe, en las viviendas donde viven ancianos. Estamos hablando de la falta de teléfono, electricidad, agua caliente y ducha o baño. A estas características se la asocia la existencia de cocina en la vivienda. Por todo ello, el tercer factor esta hablando de unas viviendas sin las instalaciones y servicios elementales, es decir, de baja calidad.

El cuarto factor también se asocia a variables de vivienda, y sólo nos explica un $7,8 \%$ de la varianza total, y está compuesto por un menor número de variables. Nos describe un tipo de vivienda ocupadas por ancianos que se por su mediano tamaño (5 habitaciones y entre 60 y $90 \mathrm{mts}$., haber sido edificadas entre los años 1960 y 1980 y pertenecer a la categoría de régimen de tenencia en propiedad. Se puede decir que este factor, más indeterminado, define a unos ancianos que poseen una vivienda de tipo medio (por edad y tamaño).

Igual se puede decir de los factores quinto $(6,4 \%$ de la varianza total) y sexto $(5,2 \%)$, relacionados fuertemente con unas pocas características de las viviendas de los mayores. El primero de ellos le hemos denominado vivienda de tipo alto 
por estar asociado equipamientos y servicios de las mismas que no se dan normalmente en las viviendas, como son el de la existencia de refrigeración, instalación de gas y calefacción individual. Al contrario ocurre con el último de los factores que engloba variables tales como un tamaño muy reducido (menos de $30 \mathrm{mts}$.), una edad muy antigua (edificadas antes de 1941) y un régimen de tenencia de alquiler, todo lo cual nos habla de viviendas de muy baja calidad. Este último factor, como apuntamos al principio de este apartado, se relacionaba en los análisis previos a la condición sociodemográfica de ancianos analfabetos, lo cual esta en consonancia con la vivienda descrita.

En conclusión podemos afirmar que los factores que definen los distintos tipos de ancianos existentes en el municipio de Sevilla en 1991 se basan en el lugar de procedencia, el nivel de instrucción y en relación con la actividad, demográficamente hablando, y en el número de ancianos residentes en cada vivienda, así como en el tamaño, el año de construcción, el régimen de tenencia y en las instalaciones y servicios, desde el punto de vista de las casas en las que habitan.

\section{TIPOLOGÍAS DE ANCIANOS EN EL MUNICIPIO DE SEVILLA}

Con los pesos obtenidos para cada sección censal de cada uno de los seis factores se realizó el análisis cluster. Este tipo de análisis nos va a permitir agrupar las secciones censales en clusters (grupos) en función de los factores anteriormente hallados. De esta forma vamos a identificar que secciones son similares o lo que es igual, aquellas que posean ancianos con las mismas características demográficas, socioeconómicas y de vivienda. El Mapa 1 muestra la distribución urbana de áreas homogéneas de la ciudad de Sevilla según los distintos grupos de ancianos que viven en ellas.

En un principio se realizó un solo cluster ya que no disponíamos información sobre el número adecuado a seleccionar. Tras varias pruebas y luego de consultar los dendogramas (Mapa 1) se optó por diez grupos ya que éstos ofrecían un adecuado nivel de diferenciación. Los centroides resultantes para cada grupo se recogen en el cuadro V. Sin embargo, y como puede verse, los cluster 7 y 10 no se pueden considerar como tales ya que sólo agrupan una y dos secciones, respectivamente, lo cual no nos parece significativo. En cuanto al resto, decir que los cluster 1 y 4 provienen del mismo grupo, así como el 2 y 3 , el 5 y 6 y el 7 y 8 . A su vez, los clusters $1,2,3$ y 4 son subgrupos originados por la subdivisión de uno anterior. Por ello se podría hablar realmente de cuatro grupos: el primero formado por los cluster 1 y 4 ; el segundo por el 2 y 3 ; el tercero por el 5 y 6 ; y el último, por el 7 y 8 . 


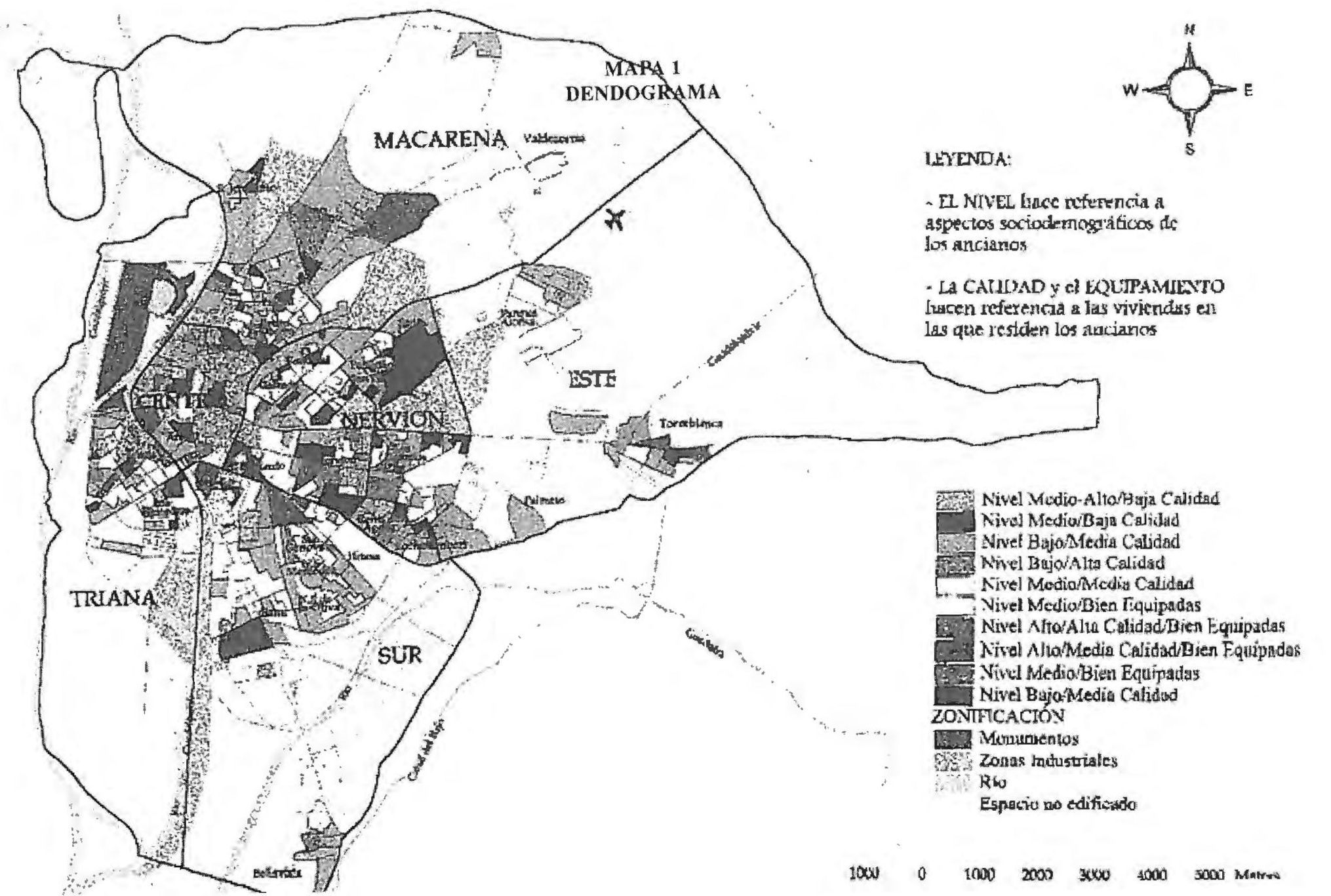


CUADRO $\mathrm{V}$

CENTROIDES DE LOS CLUSTERS

\begin{tabular}{|c|c|c|c|c|c|c|c|c|}
\hline $\begin{array}{c}\text { Cluste } \\
\text { r }\end{array}$ & $\mathrm{N}^{0}$ & $\%$ & $\begin{array}{c}\text { Factor } \\
1\end{array}$ & $\begin{array}{c}\text { Factor } \\
2\end{array}$ & $\begin{array}{c}\text { Factor } \\
3\end{array}$ & $\begin{array}{c}\text { Factor } \\
4\end{array}$ & $\begin{array}{c}\text { Factor } \\
5\end{array}$ & $\begin{array}{c}\text { Factor } \\
6\end{array}$ \\
\hline 1 & 42 & 9,5 & 4,66 & 5,09 & 5,82 & $-1,60$ & 0,17 & 0,40 \\
\hline 2 & 195 & 44,12 & $-5,15$ & $-4,07$ & $-1,28$ & $-0,68$ & $-0,48$ & $-1,12$ \\
\hline 3 & 28 & 6,33 & $-1,09$ & $-2,91$ & 0,76 & $-0,87$ & 4,69 & $-2,08$ \\
\hline 4 & 35 & 7,92 & $-1,32$ & $-3,40$ & $-1,79$ & 5,03 & $-0,42$ & $-1,15$ \\
\hline 5 & 33 & 7,47 & 8,79 & 3,06 & 0,50 & 0,69 & 1,48 & 3,11 \\
\hline 6 & 87 & 19,68 & 2,80 & 3,69 & $-0,72$ & 0,12 & $-1,08$ & 1,59 \\
\hline 7 & 2 & 0,45 & 11,82 & 0,52 & 1,67 & 0,60 & 11,36 & $-0,01$ \\
\hline 8 & 10 & 2,26 & 23,32 & 16,40 & 2,97 & $-0,34$ & 0,18 & 5,18 \\
\hline 9 & 9 & 2,04 & 11,62 & 22,19 & 7,05 & $-1,41$ & $-1,46$ & 1,03 \\
\hline 10 & 1 & 0,23 & $-11,08$ & $-7,92$ & $-3,42$ & 28,95 & 3,33 & $-1,92$ \\
\hline
\end{tabular}

Para realizar el agrupamiento de las secciones censales en el análisis cluster se utilizó el método conocido como Furthest Neighbor (amalgamamiento completo) en el que se considera que la distancia o similitud entre dos cluster hay que medirla atendiendo a sus elementos más dispares. Por tanto, la distancia o similitud entre clusters viene dada por la máxima distancia (o mínima similitud) entre sus componentes. Se trabajó con distancias métricas (por imperativo del programa escogido), concretamente con la Euclidean (euclídea) y el análisis se efectuó por variables de los seis factores.

Como puede verse el cluster 1 está formado por 42 secciones censales (que representan el 9,5\% del total de secciones). Los centroides presentan valores altos para los factores 2 (ancianos de nivel alto), 3 (ancianos de nivel medio) y 1 (viviendas de baja calidad) en este orden. Las secciones censales que integran este grupo se caracterizan tanto por la presencia de ancianos formados, activos y residentes en casas grandes, como por ancianos con poca o media formación, jubilados y viviendo en casas de tipo medio. Sin embargo, la fuerte proximidad de este cluster al tercer factor nos hace suponer que estos ancianos, independientemente de su formación, número de ancianos residentes o el tamaño de su vivienda, habitan en casas mal equipadas o de baja calidad, tal y como este factor se caracteriza. Este grupo esta compuesto por 13759 ancianos que se distribuyen por el distrito Centro, y los barrios de Nervión, Porvenir y Triana.

El cluster 4 (recordemos que junto al anterior formaban previamente un mismo grupo) esta compuesto por un número similar de secciones, 35 en concreto $(7,92 \%)$. En este grupo parece predominar la presencia de viviendas de tipo medio (debido a los altos valores respecto del factor 4) y la ausencia de ancianos de nivel alto, según lo atestigua el bajo valor del factor 2. Este cluster totaliza 8108 
personas mayores repartidas por los distritos periféricos: San Jerónimo, Polígonc San Pablo y entre los barrios de Rochelambert y Cerro del Águila.

El cluster 2 es el más numeroso y está compuesto por 195 secciones censales (lo que supone el 44,12 \% del total de las mismas). Las secciones censales que forman parte de este cluster se caracterizan por sus valores negativos respecto de todos los factores, especialmente de los tres primeros. En consecuencia podemos hallar en estas secciones un predominio de ancianos con poca formación pero residiendo en viviendas de tipo medio alto (factores 4 y 5). Este grupo es el que más repartido está de todos, junto con el 3, y encontramos este tipo de secciones en San Jerónimo, Pino Montano (distrito Norte o Macarena), Parque Alcosa, Rochelambert, Palmete (distrito Este), sector norte del distrito Centro (Alameda de Hércules y calle San Luis) y Triana.

En cuanto al cluster 3 (con 28 secciones censales, el 6,33\%) lo forma un grupo de similares características que el anterior en cuanto al predominio de ancianos de nivel medio que habitan en esas secciones censales; sin embargo, a diferencia del anterior, estos ancianos parecen que viven en casas de alta calidad, aunque también es significativa la existencia de las de calidad baja (factor 3). Es decir, existe una presencia y alternancia de viviendas de alta calidad (urbanizaciones, casas aisladas, polígonos) y zonas de construcciones propias, o quizás en algunas de estas 28 secciones predominen el primer tipo de viviendas, y en otras el segundo. Este grupo de ancianos suman un total de 6462 personas mayores y, como dijimos, están muy repartidos por toda la trama urbana de la ciudad junto al anterior grupo.

Por su parte los clusters 5 y 6 provienen de un mismo grupo. El primero (con 33 secciones, el 7,47\% de ellas) esta caracterizado por ancianos de nivel medio que residen en viviendas con equipamientos e instalaciones que denotan un buen nivel económico, como aire acondicionado, instalación de gas o calefacción. Este tipo de secciones suma un total de 12427 personas de la tercera edad que encontramos en los barrios de la Bazorla, el Fontanal, Polígono de San Pablo y Santa Clara (distrito de Nervión), Nª . S . de la Oliva (sector Sur), y Pío XII-Macarena y San Jerónimo (distrito Norte).

El grupo 6 es el segundo más numeroso con 87 secciones censales $(19,68 \%$ del total). Este grupo se caracteriza por tener un alto número de ancianos entre los que predominan la condición socioeconómica media-alta (segundo factor) residiendo también en casas bien equipadas o de alta calidad (sexto factor). Este grupo son 26367 ancianos repartidos por Los Remedios y Reina Mercedes (en torno a las Avenidas de la República Argentina y de la Palmera, respectivamente), en Hitasa Carmona (distrito Sur), Parque Alcosa (distrito Este) y en la Carretera de Carmona (distrito Norte).

Los cluster 8 y 9 , como dijimos, formaban también un único grupo antes de la última subdivisión, y debido a ello son bastante parecidos: por el número de seccio- 
nes que los componen (10 y 9, respectivamente); o por el predomino de ancianos de alto nivel sociocultural (grados de formación universitaria). Se diferencian en que en el grupo 8 las viviendas donde residen los ancianos son de tipo medio-alto, es más numeroso (5.640 ancianos) y se localiza en el barrio del Arenal, en el Plantinar-Avenida San Francisco Javier y en Torreblanca; mientras que en el 9 de tipo medio-bajo en función de las instalaciones y equipamientos, y en el año de edificación, engloba un menor número de ancianos (3.996) que se localizan en el distrito Centro, principalmente, San Bernardo, Heliópolis y en torno a la Plaza de Cuba.

Por último, el cluster 7 (formado por sólo dos secciones censales) está compuesto por 765 ancianos, mientras que la sección del grupo 10 engloba únicamente a 119 personas mayores.

En el mapa siguiente puede verse la distribución de los distintos grupos de ancianos en el municipio de Sevilla.

\section{LA ENCUESTA}

La realización de la encuesta tenía como objetivo completar la caracterización de la población anciana en Sevilla. Hasta ahora hemos trabajado sólo con variables de tipo demográfico y de vivienda que nos han servido para realizar una tipología de personas mayores en los capítulos precedentes. Sin embargo, en la elaboración de esa clasificación de ancianos no hemos podido tener en cuenta variables tan importantes de tipo económico como el nivel de renta (pensiones, ayudas, ingresos), o aspectos sobre la localización del anciano en la ciudad (dónde ha vivido, dónde vive, etc.), o cuestiones sobre condiciones de vida actuales (aspectos sobre la salud, vida cotidiana, movilidad diaria, autonomía, privacidad, seguridad, etc.), o situación de los servicios y necesidades para ellos porque carecemos de esos datos. Es por ello por lo que se plantea la realización de una encuesta, ya que estas tiene como finalidad de completar la información que no es posible encontrar en las fuentes oficiales o no está disponible al nivel de desagregación que interesa para alcanzar los objetivos de un proyecto.

En nuestro caso es muy importante contar con esta información no censal, no sólo para completar y mejorar la caracterización de la población anciana como se ha dicho, sino también y en mayor medida para poder en un futuro evaluar las necesidades de servicios sociales, asistenciales y culturales que tienen o pueden tener los mayores de Sevilla ${ }^{3}$.

3. Una vez establecida la distribución espacial de la gente mayor y sus características y tipología, la información generada con el apartado sobre servicios sociales de la encuesta será utilizada para la fase final del proyectu en el que éste forma parte. La información servirá para confrontar los recursos ofertados desde la administración y otros entes públicos y privados, con idea de diseñar un pliego sobre las demandas y necesidades existentes en las distintas unidades espaciales. Este sería un paso previo para establecer nuevas implantaciones en 
Como es del todo imposible conseguir estos datos de los casi 115000 ancianos que viven en Sevilla, se plantea la necesidad de realizar una encuesta directa a una muestra que sea representativa de todos los ancianos estudiados. Para que la información que se pretende recabar sea realmente representativa debe encuestarse a todos y cada uno de los distintos tipos de ancianos que se han identificado hasta ahora, pues cada uno aportará información diferente sobre sus características y sus necesidades. Por ello partimos de los distintos grupos de mayores que se identificaron en el anterior capítulo, seleccionando de cada uno de ellos una muestra a través de la técnica de muestreo estratificado. En este tipo de método la población está dividida en estratos o grupos antes de seleccionar el total de los encuestados. Una vez conocido el número de anciano de cada grupo, se escogerán las secciones censales en las estos se pueden encontrar, de forma que también espacialmente se encueste a ancianos de todos los distintos distritos y zonas del municipio de Sevilla. En los distritos Norte, Triana, Centro y Sur la selección de las secciones se llevó cabo de forma indiscriminada y con el objetivo de identificar a los grupos de ancianos más minoritarios.

Para calcular el tamaño de la muestra de cada grupo de ancianos (o lo que es lo mismo, el número de encuestas a realizar para garantizar que la información recogida sea factible y representativa del total poblacional) a cada uno se le aplicó la fórmula:

$$
\mathrm{n}=\left(\mathrm{Z}_{\mathrm{a} / 2} * \mathrm{~S} / \mathrm{e}\right)^{2}
$$

dónde $Z_{a / 2}$ representa el nivel de confianza ( 1 - a, que fue del $\left.95 \%\right), S$ es la desviación típica de la muestra y e es la precisión o margen de error (cuya cuantía nunca suele cifrarse por encima del $10 \%$ de la media muestral). El tamaño de las muestras y el número de encuestas totales se puede encontrar en el cuadro VI.

Las encuestas se hicieron visitando a los ancianos en sus casas y por el método de entrevista con el cuestionario preparado de antemano con el fin de evitar que las personas mayores se distrajeran y no tuvieran dificultad en entender todas las preguntas. Al principio se optó por utilizar grabadora para registrar las respuestas, pero ésta provocó una actitud a la defensiva de los ancianos que les impedía naturalidad y sinceridad, por lo que se cambió el método y se utilizó la libreta y la toma de notas sobre las contestaciones. En estas visitas también contamos con la ayuda de CÁRITAS. Por lo general se efectuaron por la tarde, horario más adecuado para encontrar al encuestado en su domicilio teniendo en cuenta las fechas en las que se realizaron (el mes de noviembre, ya metidos en invierno).

servicios sociales y la mejorar la distribución espacial de los mismos en la ciudad de Sevilla. Para esta comparación se utilizará la información de las encuestas en este aspecto, los objetivos previstos por el Plan Gerontológico y las actuaciones llevadas a cabo en otras ciudades y comunidades autónomas, con idea de establecer las posibles actuaciones en materia de asistencia y ayuda para la población anciana y la ubicación de las mismas en la ciudad de Sevilla. 
CUADRO VI

TAMAÑO DE LAS MUESTRAS DE CADA TIPO DE ANCIANOS

\begin{tabular}{lrlcc}
\hline & Población & Media & Desviac. Estand. & № de Encuestas \\
\hline Grupo 1 & 13.759 & 327,6 & 64,93 & 15 \\
Grupo 2 & 37.326 & 191,42 & 57,08 & 34 \\
Grupo 3 & 6.462 & 230,79 & 82,13 & 49 \\
Grupo 4 & 8.108 & 231,66 & 71,83 & 37 \\
Grupo 5 & 12.427 & 376,58 & 61,68 & 10 \\
Grupo 6 & 26.367 & 303,07 & 57,92 & 14 \\
Grupo 7 & 5.640 & 564 & 48,09 & 3 \\
Grupo 8 & 3.996 & 444 & 89,59 & 16 \\
Total & 114.085 & - & - & 178 \\
\hline
\end{tabular}

Los resultados obtenidos, en lo que se refiere a las características generales de tipo demográfico y de vivienda, la encuesta arrojó unos resultados medios muy parecidos a los obtenidos del Censo, destacando que:

- La edad media de los ancianos encuestados fue de 70, predominando los grupos de edad entre 60-64 y 65-69 años (el 52\% de todos los ancianos visitados).

- La mayoría de los encuestados son casados (en un 53\%) o solteros (en un $26 \%$ de los casos).

- En cuanto al nivel de instrucción, el 20\% de los encuestados no tienen estudios, el $40 \%$ tienen Grado 1, el 21\% Grado 2 y un 13\% estudios universitarios.

- El $33 \%$ de los ancianos son activos (de éstos el 20\% sobrepasan la edad de jubilación y otro $20 \%$ son voluntarios), y el resto está jubilado. De los primeros, ninguna es mujer, aunque de éstas un 33\% están jubiladas laboralmente y otro $38 \%$ trabajaron en su juventud (normalmente antes de casarse).

- La mayoría de las viviendas en las que residen los ancianos encuestado se edificaron entre 1961 y 1980 (53\%), seguidas de las construidas entre 1941 y $1960(21 \%)$.

- El $80 \%$ de las casas son de propiedad de los mayores que viven en ellas, y sólo un $14 \%$ son de alquiler.

- En cuanto al tamaño, el $20 \%$ tienen 3 o menos habitaciones, el $53 \%$ entre 4 y 5 , y el $27 \%$ restante son de 6 y más habitaciones. 
- Los ancianos viven en por lo general con otra persona mayor (53\% del to. tal), que en el $75 \%$ de los casos es la pareja, el $20 \%$ solos, y el $5 \%$ has tres ancianos conviviendo en la misma casa.

- Por último, el $47 \%$ de las viviendas tienen ascensor, el 20\% calefacción, e $100 \%$ agua caliente y el $93 \%$ teléfono.

Si atendemos al primero de los objetivos que se proponía la encuesta, es decir a generar una información sobre aspectos sobre la localización y movilidad de los ancianos en la ciudad o cuestiones sobre condiciones de vida actuales, hemos obtenido la siguiente información:

- La mayoría de los encuestados son inmigrantes (el 80\%) y hace una medic de 29 años que llegaron a la ciudad (en 1968).

- De éstos sólo el 8\% vino para buscar trabajo, mientras que el resto, en el caso de los hombres y las mujeres que tenían un trabajo, emigraron po1 motivos laborales (las respectivas mujeres, por motivos familiares).

- Estas ancianos presentan un alto porcentaje de movilidad intraurbana ya que el $60 \%$ de ellos vivió antes en otra casa.

- Los motivos por los que los ancianos se cambiaron de domicilio fueron: por una casa mayor $(22 \%)$, por tener una casa mejor (34\%), por ruina o desalojo $(11 \%)$, por tener una casa en propiedad $(20 \%)$, y por motivos familiares $(13 \%)$.

- Sólo un $13 \%$ de los encuestados está descontento con su vivienda. Sin embargo sólo el $7 \%$ lo está con su barrio.

- El 77\% de los ancianos jubilados (el resto tiene como ocupación diaria su trabajo) ocupan su tiempo principalmente en hobbies (el $81 \%$ del total) entre los que destacan: la lectura (43\%), actividades culturales como teatro y música $(29 \%)$ o en tareas relacionadas con su antiguo trabajo (14\%); cuando no se dedican a los hobbies emplean el tiempo en ir de compras o hacer recados $(73 \%)$, pasear y estar con la familia ( $45 \%$ respectivamente), acudir a algún centro de ocio o tercera edad (27\%) y hay un caso, ya mencionado, que trabaja como voluntario en CÁRITAS.

- Respecto al importante tema del nivel económico o ingresos, que determina en gran parte, como luego veremos, el nivel de vida, el $19 \%$ de los que cobran jubilación esta está por debajo de las 50.000 pts., el $45 \%$ entre 50 y 75.000 pts., un $18 \%$ entre 100 y 150.000 pts. y el $18 \%$ restante más de 150.000 pts. Sin embargo, si se analizan los ingresos por sexos, las diferencias se acentúan: mientras que sólo el $27 \%$ de todas las pensiones recaen sobre hombres, éstos son los únicos que reciben pensiones superiores a 150.000 pts. no encontrando en este grupo a ninguna mujer (sólo hay un hombre con una pensión entre 50 y 75.000 pts.); sin embargo, sólo el 13\% 
de las mujeres tienen una pensión comprendida entre 100 y 150.000 pts., mientras que el 87 restante reciben menos de 100.000 , aunque realmente se puede decir que más del $55 \%$ de ellas reciben en torno al las 55.000 pts. que supone la pensión por viudedad, que es la mayoritaria entre las mujeres ancianas. En cuanto a los ancianos que trabajan, es difícil saber sus ingresos actuales ya que la mitad llevan negocios propios (empresarios que no emplean en el sector servicios), mientras que la otra mitad son funcionarios públicos.

Por último, respecto a la información recogida referente a los servicios asistenciales y culturales las preguntas de este apartado han tratado de identificar los servicios que reciben, necesitan y demandan los ancianos. Para ello se ha hizo una primera distinción conceptual entre servicio y necesidad: el primero hace referencia a un deseo (como pueden ser actividades de ocio) y se derivan de la salida del anciano del mercado de trabajo y por tanto, de la necesidad de llenar el tiempo libre; mientras que el segundo hace referencia a una necesidad propiamente dicha o asistencia (como es el caso de ayuda domiciliaria), provocada por lo general por algún tipo de incapacidad ${ }^{4}$. Teniendo en cuenta esta distinción, hemos obtenido la siguiente información sobre servicios recibidos:

- Todos los ancianos encuestados tienen régimen de Seguridad Social y por tanto cubiertas sus necesidades de salud y asistencia médica (incluso el $20 \%$ de los encuestado tienen, además, algún seguro rnédico en compañía privada).

- Hay un $26 \%$ de ancianos que reciben ayuda domiciliaria y, otro tanto, ayuda económica por parte de CÁRITAS. Esta ayuda se ofrece a ancianos discapacitados físicamente o impedidos, normalmente solos que no pueden valerse por si mismos para las tareas más básicas: comida, aseo, movilidad. Estas ayudas provienen de unos programas anuales aprobados y financiados por el Ministerio de Asuntos Sociales y gestionados por CÁRITAS. Estas ayudas comprenden unas becas para el complemento de las pensiones (en el $95 \%$ de los casos, en torno a las 50.000 pts.), parte de la cual se emplea en pagar a una persona (en paro, con necesidades económicas) para que ayude al anciano/a en esas tareas básicas en las que no puede valerse por si mismo. Esa persona acude al domicilio del becado de dos a tres veces por semana un par de horas al día. También se prestan ayudas para mejora en las viviendas de estos ancianos incapacitados (en un 30\% de los casos encuestados), por lo general en la adaptación del baño y la cocina.

4. Así, por ejemplo, el Plan Gerontológico plantea dentro de sus objetivos el ofrecer unos servicios sociales ... para dar respuesta a las necesidades de las personas mayores, de igual modo que se propone el facilitar el acceso de las personas mayores a los bienes culturales y fomentar el empleo creativo del ocio y del tiempo libre (PLAN GERONTOLÓGICO,1993). 
- Ante la pregunta ¿Qué necesidades tiene?, un $20 \%$ de los ancianos encuestados confesó no tener ninguna, mientras que en un $47 \%$ la necesidad mayor se centra en aspectos de salud y de compañía; un $33 \%$ manifestaba necesitar actividades de ocio y cultura; para un $27 \%$ de los encuestados sus preferentes necesidades se centraban en aspectos económicos y de vivienda; y sólo un $13 \%$ de los encuestados tenía necesidades referidas a la mejora de su educación o formación y de movilidad.

- Contrasta con las anteriores respuestas que sólo el $27 \%$ de los ancianos encuestados (la totalidad de los que reciben becas del Ministerio) confesó necesitar algún tipo de servicios.

- En cuanto a sí asisten con regularidad a alguna asociación para mayores o centro de ocio, excluyendo a los que aún están activos, el $20 \%$ respondió que No porque no le gustan, mientras que un $40 \%$ de los encuestados van $o$ han ido a este tipo de asociaciones (Casino Militar o al Centro del Distrito correspondiente, en la mayoría de los casos). Sólo un 7\% de los ancianos preguntados dijo conocer asociaciones para la tercera edad.

\section{CONCLUSIONES}

En este último apartado vamos a intentar sintetizar todos los resultados de los análisis estadísticos realizados en los anteriores puntos. De esta forma vamos a ver que aquella primera idea general de cómo es el anciano del municipio de Sevilla (que se desprendía del estudio de sus principales características de demográficas y de vivienda), se va a transformar en diferentes tipos de ancianos. Los principales rasgos de éstos aparecen definidos no sólo a partir los análisis factorial y cluster, sino también por las asociaciones y las correlaciones existentes entre las variables demográficas y de vivienda estudiadas. También veremos que los distintos grupos de ancianos no se distribuyen homogéneamente por la trama urbana, sino que en la misma sección censal podemos encontrar varios tipos de ancianos, y que esto responde, en gran medida, al desarrollo urbano de la ciudad y al pasado laboral (y en consecuencia, a la formación y cualificación) de los ancianos. Será esto último, precisamente, lo que pone de manifiesto el porqué cada uno de los diferentes tipos de ancianos demanda o recibe unos servicios u otros, y cuáles van a demandar en un futuro próximo.

\subsection{Los distintos grupos de ancianos}

Al analizar las distintas características de demográficas y de vivienda de la población de 60 y más años en Sevilla en 1991, junto con las correlaciones existentes entre ellas y según las conclusiones obtenidas del análisis factorial, se pue- 
de decir que la variable viviendas principales según el número de ancianos residentes constituyen el rasgo determinante para establecer los distintos grupos de ancianos con las mismas características debido a que esta variable presenta fuertes relaciones tanto con los distintos grupos de variables demográficas, como con los de viviendas.

Las distintas categorías posibles de viviendas principales según el número de ocupantes, tal y como hemos visto, se quedaban reducidas a sólo tres para que fuesen más significativas: las viviendas con 1 ocupante, viviendas con 2 ocupantes y viviendas con 3 y más ocupantes. En función de cada una de ellas obtenemos tres principales grupos distintos de ancianos, cada uno íntimamente asociado a cada una de esas tres categorías, que a su vez se subdividen en dos, por lo que al final tenemos los seis grupos que el análisis cluster nos ofrecía.

\section{Grupo A}

El primero de los grupos de ancianos son aquellos que residen en viviendas principales ocupadas al menos por un anciano. Se puede decir, por tanto, que este anciano tiene un núcleo familiar reducido.

Sus características demográficas más importantes son: predominan los viudos, y en menor medida solteros y separados o divorciados; tienen entre 60 y 80 años y son ancianos sin estudios o con el primer grado; por lo general están inactivos aunque también destacan los parados; entre los activos y ocupados destacan los que trabajan en el sector de la industria; y residen en viviendas principales. Dicho de otro modo, la presencia del primer factor que denominamos ancianos de nivel medio, es muy predominante

Como vimos al tratar las correlaciones, la asociación entre la edad, el estado civil y el nivel de instrucción indicaba que los viudos o solteros tienen una menor edad y un menor nivel de instrucción. A su vez, la relación existente entre el nivel de instrucción, la actividad y el sector de ocupación indica que a menor grado de estudios, mayor inactividad y una cierta relación con la ocupación el sector secundario. Por tanto se puede deducir que tenemos dos subtipos de ancianos que residen en viviendas principales ocupadas por un anciano:

a) Unos son viudos o solteros, tienen por lo general entre 60 y 75 años, no tienen estudios, pueden estar en paro o inactivos y los que no lo están, trabajan en la industria.

b) Otros son separados o divorciados, tienen más edad que los anteriores (entre 70 y 80 años), tiene el primer grado, y o son inactivos o trabajan en el sector industria. 
Por otro lado, las principales características de las viviendas que ocupa este tipo de anciano se agrupan sobre la base de la importante correlación existente entre el tamaño de las viviendas (y consecuentemente con el número de habitaciones), el año de construcción y determinado régimen de tenencia de las mismas. Así tenemos viviendas con menos de 60 metros (de 2 a 4 habitaciones, por tanto), que datan de antes de 1961 (y son viviendas en alquiler) o de 1981 en adelante (y entonces son viviendas en propiedad). Además estas viviendas están en edificios de 3 y más viviendas. Por todo ello se deduce que las viviendas de estos ancianos están muy determinadas por los factores vivienda de baja y media calidad, o lo que es lo mismo, con los factores 4 y 6 .

En definitiva se puede concluir que este grupo esta formado por los ancianos de nivel medio que residen o en viviendas de baja calidad o de calidad media, y viven en un núcleo familiar pequeño o incluso solos, son viudos, solteros, separados o divorciados, tienen una edad joven por lo que aún mantienen un alto grado de autonomía física o movilidad y de salud, tienen pocos estudios, y pueden estar prejubilados o jubilados o trabajar en actividades manufactureras. Viven en viviendas principales, de pequeño tamaño y pocas habitaciones, que si son viejas (edificadas antes de 1941) pueden ser alquiladas de renta antigua, y si son de reciente construcción son de su propiedad.

\section{Grupo B}

El segundo de los grupos de ancianos son aquellos que residen en viviendas principales con dos ocupantes.

Las características demográficas más importantes de estos ancianos son: predominan los solteros, y en menor medida viudos y casados; tienen también entre 60 y 80 años y son ancianos sin estudios, con el primer o segundo grado; están tanto inactivos como activos (y por tanto ocupados) trabajando en los sectores de la industria o de los servicios; y, en cuanto a las viviendas en las que residen, generalmente son principales, aunque también hay ancianos en colectivas. Por tanto estamos hablando una importante influencia del factor 1 o ancianos de nivel medio.

De igual manera que para el caso anterior, la relación entre la edad, el estado civil y el nivel de instrucción nos indicaba que los solteros tienen una menor edad y un menor nivel de instrucción, y los casados más años y más estudios. A su vez, la relación existente entre el nivel de instrucción, la actividad y el sector de ocupación indicaba que a menor grado de estudios, mayor inactividad, y si existían ancianos ocupados, predominaba el sector secundario, mientras que a mayor grado de estudios, mayor actividad y ocupación en los servicios. Por tanto se puede deducir que también tenemos dos subtipos de mayores que residen con otro anciano: 
a) unos son solteros, tienen por lo general entre 60 y 70 años, tienen el primer grado o no tienen estudios, están inactivos y los que no lo están, trabajan en la industria.

b) otros son casados, tienen más edad que los anteriores (entre 70 y 80 años), tiene el segundo grado o el primero, y ancianos activos que trabajan en el sector servicios.

Por otro lado, los rasgos más importantes de las viviendas ocupadas por estos ancianos tienen una importante relación con el tamaño de las viviendas (y consecuentemente con el número de habitaciones), con el año de construcción y con determinado régimen de tenencia de las mismas. En consecuencia, las viviendas de los ancianos que con dos ocupantes también son de menos de 60 metros (pero sin embargo tienen de 3 a 5 habitaciones), datan de antes de 1961 o de 1981 en adelante (y son viviendas en propiedad). También estas viviendas con dos ocupantes están en edificios de 3 y más viviendas. En definitiva nos estamos refiriendo a ancianos que residen en viviendas de tipo medio y viviendas bien equipadas o tipo alto (factores 4 y 5 ).

Por todo ello se puede concluir que los ancianos del grupo B son aquellos de tipo medio cuyas viviendas son también de tipo medio e incluso alto. Estas personas mayores viven con otra persona, y son solteros, viudos o casados. Los dos primeros viven con otro familiar, que también puede ser anciano, tienen una edad joven y aún mantienen un alto grado de autonomía física o movilidad y de salud, tienen pocos estudios, y pueden estar prejubilados o jubilados o trabajar en actividades manufactureras. Los ancianos casados que habitan casas con dos ocupantes viven con su cónyuge, presentan más edad (lo puede que limitar su autonomía y salud), y han cursado el bachillerato, trabajando en algún negocio familiar. Ambos, viudo o solteros y casados, Viven en viviendas principales, de pequeño tamaño pero con más habitaciones que los ancianos que viven solos, que tanto si son antiguas o de reciente construcción, son de su propiedad.

\section{Grupo C}

El tercer grupo de ancianos lo constituyen aquellos que residen en viviendas principales con tres y más ocupantes. Las características demográficas más importantes de estos ancianos es que son principalmente solteros y casados, tienen también más de 80 años, son de segundo grado, están activos (y por tanto ocupados) trabajando en los servicios. En cuanto a las viviendas en las que residen, generalmente son principales aunque también hay ancianos en viviendas colectivas, por lo que el núcleo familiar tiende a ser de un importante número de personas. 
En este caso, también la relación entre la edad, el estado civil y el nivel de instrucción nos indica que los casados tienen más edad, y a mayor edad mayor nivel de instrucción. A su vez, la relación existente entre el nivel de instrucción, la actividad y el sector de ocupación indicaba que a mayor grado de estudios, mayor actividad (ocupación) y mayor relación con el sector servicios. Por tanto se puede deducir que el tipo de ancianos que viven con más de dos personas (que residen en viviendas principales con tres y más ocupantes) responde a un perfil de anciano casado (en menor grado soltero), que tiene más edad que los anteriores (80 y más años), han cursado el segundo grado de estudios (y algunos, estudios universitarios), y son ancianos activos que trabajan en el sector servicios. En definitiva, estamos hablando del segundo factor o ancianos de nivel alto.

Por otro lado, también vimos que las viviendas principales ocupadas por tres y más ocupantes tienen una importante relación con el tamaño de las viviendas, con el número de habitaciones y con el régimen de tenencia. En consecuencia, las viviendas de los ancianos que con tres y más ocupantes son de más 91 metros (tienen más 5 habitaciones), datan de entre 1941 y 1961, y son viviendas en propiedad, lo cual esta en consonancia el con el tamaño del núcleo familiar en relación con un mayor número de personas mayores conviviendo en las misma casa.

Por todo ello se puede concluir que los ancianos del grupo $\mathrm{C}$ son ancianos con un nivel alto en sus condiciones de vida y vivienda, que se caracteriza ante todo por su tamaño. Estos ancianos viven con su familia, y son casados y solteros. Los primeros presentan una edad más avanzada que los segundos (lo puede que limitar su autonomía y salud), y han cursado el bachillerato e incluso han ido a la universidad y trabajan en algún negocio familiar. Viven en viviendas principales de gran tamaño y con muchas habitaciones, construidas al menos hace 30 años y son de su propiedad.

\subsection{Consideraciones sobre la planificación de servicios sociales y asistenciales para los ancianos del municipio de Sevilla}

Uno de los objetivos principales de la encuesta era que sirviese de base para una futura planificación de servicios para la tercera edad. De los resultados obtenidos, aflora una consecuencia inmediata: las preguntas y las respuestas sobre los servicios y necesidades que éstos tienen o demandan mejoran la caracterización que definen a los distintos grupos de ancianos, así como la comprensión de ellos. Y esto es así porque lo demandado o necesitado está en función de la formación, la cualificación y la actividad laboral de la persona mayor, o lo que es lo mismo, del tipo de anciano que sea. Junto a esto, además hemos constatado que el lugar de residencia también viene determinado por estas características, a la que habría que sumar el origen de las personas mayores. Si tenemos en cuenta que, luego de 
la aplicación de las distintas técnicas estadísticas (descriptivas y multivariantes), los grupos de ancianos que hemos identificados en este proyecto no son del todo homogéneos (lo cual dificultaría una posible actividad de planificación), las conclusiones de la encuesta revelan la importancia de este método para conseguir alcanzar nuestro objetivo de identificar grupos de ancianos homogéneos y distintos unos de otros, estadística y espacialmente.

En efecto, luego del desarrollo completo de este proyecto, para poder plantear una planificación de servicios para la tercera edad en el municipio de Sevilla hace falta profundizar más en los aspectos espaciales, es decir, romper las barreras que imponen las secciones censales que permitan identificar clusters (grupos de ancianos) homogéneos y bien definidos, porque así es como se llegaría a identificar unitariamente los grupos de distintos tipos de ancianos en cada parte de la ciudad, ya que hasta ahora, y con la metodología aplicada, sólo es posible llegar a saber que en un área espacial amplia (con una escala demasiado grande cuando estamos hablando de caracterizar personas y sus viviendas) coexisten distintos grupos de mayores, pero sin poder precisar más, espacial y sociodemográficamente. Esto sería posible, a nuestro entender, por una doble vía:

a) una elaboración de la información censal más detallada; y

b) un estudio más profundo en una región urbana concreta, analizando con detalle todas y cada una de las características demográficas, sociales, económicas, de vivienda y relativas a los servicios y necesidades que este proyecto ha puesto de manifiesto como definitorias de las distintas tipologías de anciano, apoyándose en una encuesta más amplia y numerosa.

\section{BIBLIOGRAFÍA}

BASULTO, y ARIAS: «Un estudio de la diferenciación residencial en el espacio urbano de Sevilla». Cindad y Territorio, 1989 (79).

BODERO AZCANO, M.F. y C. CRESPO TOBARRA: «Bibliografía sobre Tercera Edad». Boletín de Estudios y Documentación de Servicios Sociales, 1984 (18-19): 87-104.

BOSQUE MAUREL, J., FERNÁNDEZ GUTIÉRREZ, F, BOSQUE SENDRÁ, J. y PÉREZ

ALCAIDE, F.: Atlas social de la ciudad de Granada. Edita Caja General de Ahorros de Granada, 1991.

BOSQUE SENDRÁ, J.: Sistemas de Información Geográfica. Rialp, Madrid 1992.

BOSQUE SENDRÁ, J., CEBRIÁN DE MIGUEL, J.A., y OTROS: Aplicaciones de la Informá-

tica a la Geografía y las Ciencias Sociales. Editorial Síntesis, Madrid 1988.

CAMPO MARTÍN, A.: "Una aplicación de la ecología factorial al estudio de pautas espacia-

les». Ciudad y Territorio, 1983 (58): 137-145.

«La segregación social en el municipio de Madrid». Ciudad y Territorio, 1985 (14).

CÁRITAS DIOCESANA DE ZARAGOZA: Estudio sociológico sobre las personas mayores de

65 años. Departamento de Ancianos, Zaragoza, 1991. 
CÁRITAS DIOCESANA DE ZARAGOZA: Informe sociológico sobre las personas mayores de 60 años en el Distrito II de Zaragoza. Departamento de Ancianos, Zaragoza, 1991.

CÁRITAS DIOCESANA DE LOGROÑO: Estudio sociológico de las personas mayores de 65 años en La Rioja. Cáritas Diocesana, Logroño, 1991.

CENTRO DE INVESTIGACIONES SOCIOLÓGICAS: Situación social de los viejos en España. Estudios y Encuestas, 1990, (21): 3-100.

CENSO DE POBLACIÓN Y VIVIENDAS 1991: Instituto de Estadística de Andalucía, Sevilla, 1992.

COMUNIDAD ECONÓMICA EUROPEA: «Resoluciones y dictámenes de la Comunidad Económica Europea en materia de Servicios Sociales». Rev. de Servicios Sociales y Política Social, 1987 (7): 52-61.

CRUZ VILLALÓN, J. (Coord.): La población de Sevilla. Ayuntamiento de Sevilla, Sevilla, 1986 DEL CAMPO MARTÍN, A.: «Una Aplicación de ecología factorial al estudio de pautas espaciales de segregación social en el municipio de Madrid». Ciudad y Territorio, 1983 (58): 137 153.

ESRI-ESPAÑA GEOSISTEMAS SA: Arcópolis. Callejeros Digitales para Aplicaciones Profesionales, Manual del Usuario. Madrid, 1995.

ESRI: Usisng ARCVIEW GIS. Manual del Usuario. Madrid, 1996.

ESTÉBANEZ, F. y BRADSHAW, E.: Técnicas de Cuantificación en geograffa». Albacete, 1979. FERNÁNDEZ-BALLESTEROS, R.: «Indicadores de salud en ancianos institucionalizados». Actas del VI Congreso Nacional de Gerontología, Alicante, 1982, 12 p.

FUNDACIÓN CAJA DE MADRID: Estudio sobre las necesidades sociosanitarias de las personas mayores en el Municipio de Móstoles. Fundación Caja de Madrid, Madrid, 1994.

GARCÍA NINET, J.I.: «Bibliografía española sobre Jubilación y Tercera Edad.» Rev. Española de Seguridad Social, 1982 (16): 453-459.

GAYMU, J.: «Les populations agees en France, au recensement de 1982», Population, vol, 40, n. 4-5, 1985, pp. 699-724.

GOLANT. S.M.: «The residential location and spatial behaviour of the elderly», Dept. of Geography, Univ, of Chicago, Research Paper, n. 143, 1972.

«Residential concentrations of the future elderly», The Gerontololgist, n. 15, 1975, pp. 16-23.

INSTITUTO DE ESTADÍSTICA DE ANDALUCÍA: Atlas Comercial de Andalucía 1994. Editado por el Instituto de Estadística de Andalucía, Sevilla, 1995.

JORDÁ BORRELL, R., LUCENDO MONEDERO, A.L.: "Georreferenciación y caracterización de la población anciana de la ciudad de Sevilla». En las Actas del VII Coloquio de Geograffa Cuantitativa, Vitoria, 1996.

JORDÁ BORRELL, R., LUCENDO MONEDERO, A.L.: «Caracterización de la población anciana de la ciudad de Sevilla mediante el análisis cluster». Comunicación presentada en el VI Congreso de la Población Española, Huelva, 1997.

JUNTA DE ANDALUCÍA: Guía de Equipamientos y Servicios para la Tercera Edad de la Provincia de Cádiz, 1982.

JUNTA DE ANDALUCIA: Plan Gerontológico: Política Integral para Mayores. Borrador. Consejería de Asuntos Sociales, Junta de Andalucía, 1991.

JUNTA DE ANDALUCÍA: Jornadas Técnicas sobre Mapas de Servicios Sociales. I.E.N.D.L.C.P.S. Madrid, 1987.

KANE, R.A., y KANE, R.: Evaluación de las necesidad en los ancianos. Guía práctica de los instrumentos de medición. Caja de Madrid, Edita SG Editores, Barcelona, 1993.

LEONARDO AUTENEXTES, S.: Estructura urbana y diferenciación residencial. El caso de Bilbao. 1989. 
LEY DEL CONSEJO ARAGONÉS DE LA TERCERA EDAD DE 1990. Boletín Oficial de Aragón, 1991.LIZANA RIVAS, E.: Distribución y comportamientos espaciales de la población envejecida en Madrid. Tesina presentada en la Universidad Complutense de Madrid, 1985, pp. 174.

LÓPEZ JIMÉNEZ, J.J.: «Áreas sociales y población anciana en el municipio de Madrid: aplicación del análisis factorial a un espacio urbano diferenciado». Revista de Economía y Sociedad, 5, Revista Regional CCAA, Madrid, 1991, pp. 79-94.

- «Envejecimiento, tamaño demográfico y sector de actividad en los municipios españoles». Revista de Estudios Territoriales, 1991 (36).

— El envejecimiento y las personas ancianas de Madrid. Editado por el Área de Servicios sociales del Ayuntamiento de Madrid, 1993

LUCENDO MONEDERO, A.L, JORDÁ BORRELL, R., y PANOFF, R.: «Future and posibility of the GIS application to the study of the desabled populations. The case of the elderly in Seville». GIS World, 1997 (en prensa).

MENÉNDEZ DE LUARCA, M.: «Análisis de datos y encuestas culturales». Análisis e Investigaciones Culturales, 1982 (13): 83-105.

MORAGAS MORAGAS, R.: «Gerontología Social: envejecimiento y calidad de vida». Madrid, CINDOC, 1991

MINISTERIO DE ECONOMÍA Y HACIENDA: Simposio Internacional sobre Tendencias demográficas y planificación económica. Actas y Debates, Coord. A. Olano, Centro de Publicaciones, Documentación y Biblioteca, Madrid, 1986, 418 p.

NEWCOMBE, C.: "Graphic representation of age and sex distribution of population in the city», en Cities and Society, editores: Hatt y Reiss, 1961, Ed. Glencoe Illinois.

OCAÑA OCAÑA, C.: Estructuras Sociodemográficas y áreas sociales en la ciudad de Málaga. Consejería de Obras Públicas y Transportes. Junta de Andalucía. 1988.

PANIAGUA MAZORRA, A.: «Causas y tipologías de la tercera edad en Madrid». Revista Alfoz, 1985.

RASO, J.M., MARTÍN VIDE, J. y CLAVERO, P.: Estadística Básica para Ciencias Sociales. Edita Ariel Geografía, Barcelona, 1987.

ROBINSON, A.H., SALE, R.D., MORRISON, J.L., MUEHRCKE, P.C.: Elementos de Cartograffa. Edita Omega, Barcelona, 1987.

ROWLES, G.D.: «Prisioners of space? Exploring the geographical experience of older people». Westview, 1978, Boulder, Colorado, $216 \mathrm{pp}$.

«The geography of ageing and the aged: toward an integrate perspective». Progress in Human Geography, 1986, vol. 10, n 4: 511-539.

SANTOS PRECIADO, B.: «Dinámica demográfica de la aglomeración demográfica madrileña». Estudios Territoriales, 1991 (37).

SMITH P.D., y WILLIAMS, S.: Gentrification of the City. Edita RGH, Londres, 1986.

STIRNER, F.W.: «The transportations needs of the elderly in a large urban environment», The Gerontologist, n. 18, 1978, pp. 207-211.

TORRES SALVADOR, A.: « Los Servicios Sociales en la Comunidad Autónoma Valenciana». Rev. de Servicios Sociales y Política Social, 1987 (7): 33-35.

TRESSERRA, M.A.: «Servicios Sociales para Ia Tercera Edad: tendencias y perspectivas». Rev. de Servicios Sociales y Política Social, 1989 (14): 14-17.

TRUYOL WINTRICH, I. y A. MARTÍN LÓPEZ: «Análisis sobre la protección social a la Tercera Edad». Rev. Española de Seguridad Social, 1980 (7): 111 - 163.

URIEL JIMÉNEZ, E.; Análisis de datos. Series temporales y Análisis multivariante. Editorial AC, Madrid, 1995. 